\title{
Title of the Article-Intestinal Gas
}

\author{
Premashis Kar $^{1 *}$ and Sushil Kumar Sharma ${ }^{2}$ \\ ${ }^{1}$ Department of Gastroenterology and Hepatology, Max Super Speciality Hospital, Vaishali, UP, India \\ ${ }^{2}$ Department of Gastroenterology and Hepatology, Max Super Speciality Hospital, Vaishali, UP, India \\ *Corresponding Author: Premashis Kar, Department of Gastroenterology and Hepatology, Max Super Speciality Hospital, Vaishali, UP, \\ India.
}

Received: July 26, 2019; Published: August 14, 2019

\begin{abstract}
Intestinal gas is mainly composed of nitrogen,ooxygen,carbon dioxide,hydrogen and methane.Various complaints attributed to it are belching, flatulence abdominal bloating and abdominal distension. In patients with isolated symptoms of excessive belching, psychological and behavioral factors play an important role..Belching is also associated with gastroesophageal reflux disease (GERD) and functional dyspepsia.We should always rule out alarm features in evaluation of patients such as weight loss,bleeding per rectum,nocturnal abdomen pain diarrhoea and investigate further. Rome IV criteria for establishing the diagnosis of functional bloating should be used. Manangement of various disorders due to intestinal gas include education, treatment of associated disorders, behavioral therapy, dietary modifications and drugs.
\end{abstract}

Keywords: Intestinal Gas; Belching; Flatulence; Abdominaldistension; Bloating

\section{Abbreviations}

N2: Nitrogen; 02: Oxygen; C02: Carbon Dioxide; H2: Hydrogen; CH4: Methane; GERD: Gastroesophageal Reflux Disease; FODMAPS: Fermentable Oligosaccharides Disaccharides Monosaccharides and Polyols

\section{Introduction}

Gastrointestinal complaints are commonly attributed by the patient to gas. Excessive intestinal gas occur due to excessive air swallowing, increased intraluminal production from malabsorbed nutrients, decreased gas absorption due to obstruction or dysfunctional gas clearance, or expansion of intraluminal gas due to changes in atmospheric pressure.

Volume and composition of intestinal gas

The volume of gas in the intestinal tract is approximately 200 $\mathrm{mL}$ in both the fasting and postprandial states in both normal sub- jects and individuals who complain of gaseous distention [1]. Nitrogen $\left(\mathrm{N}_{2}\right)$, oxygen $\left(\mathrm{O}_{2}\right)$, carbon dioxide $\left(\mathrm{CO}_{2}\right)$, hydrogen $\left(\mathrm{H}_{2}\right)$, and methane $\left(\mathrm{CH}_{4}\right)$ account for more that 99 percent of expelled intestinal gas [1]. The composition of gas within the intestinal tract is predominantly $\mathrm{N}_{2}[1]$. Oxygen is present in very low concentrations, and the concentrations of $\mathrm{CO}_{2}, \mathrm{H}_{2}$, and $\mathrm{CH}_{4}$ vary among individuals. Sources of intestinal gas

Air swallowing

Aerophagia is the major source of stomach gas. Larger amounts are swallowed when food is gulped. Aerophagia can be a manifestation of anxiety, and is increased with gum chewing and smoking. Most swallowed air appears to be eructated [2]. Swallowed air is the major source of $\mathrm{O}_{2}$ and $\mathrm{N}_{2}$ in intestinal gas. Posture may influence the amount of swallowed air passing from the stomach into the small intestine. The supine position causes gastric air to pass

Citation: Premashis Kar and Sushil Kumar Sharma. “Title of the Article-Intestinal Gas”. Acta Scientific Gastrointestinal Disorders 2.7 (2019): 16-20. 
preferentially into the small intestine as gastric air is located above liquid gastric contents that overlie the gastroesophageal junction.

\section{Intraluminal production}

Three of the five principal gases, $\mathrm{CO}_{2}, \mathrm{H}_{2}$, and $\mathrm{CH}_{4}$, are produced within the bowel lumen.

- $\quad \mathbf{C O}_{2}: \mathrm{CO}_{2}$ is derived from digestion of fat and protein in the upper gastrointestinal tract, from bacterial fermentation of intraluminal substrates, or may be liberated from the interaction of acid and bicarbonate [3]. Much of the $\mathrm{CO}_{2}$ produced in the upper small intestine is probably absorbed before it reaches the colon. $\mathrm{CO}_{2}$ that is present in flatus is likely due to bacterial fermentation reactions in the colon. Increased $\mathrm{CO}_{2}$ in flatus can result from specific dietary substrates, such as nondigestible carbohydrates [4].

- $\quad \mathbf{H}_{2}: \mathrm{H}_{2}$ is both produced and consumed by fecal bacteria; the net $\mathrm{H}_{2}$ excretion is determined by these two processes. $\mathrm{H}_{2}$ production occurs predominantly in the colon [5]. Ingested carbohydrate and protein are sources for $\mathrm{H}_{2}$ production. In healthy individuals, certain foods with high concentrations of oligosaccharides found in legumes, or resistant starches (flours made from wheat, oats, potatoes, and corn), cannot be completely digested by enzymes within the normal small bowel, leading to increased $\mathrm{H}_{2}$ production [6].

- Methane: Methane, like $\mathrm{H}_{2}$, is exclusively a product of bacterial metabolism. The presence of bile acid in the colon also may be important in the regulation ofmethanogenesis [7].

- Causes of increased intestinal gas: When excessive intestinal gas occurs, it may be due to excessive air swallowing, increased intraluminal production from malabsorbed nutrients, decreased gas absorption due to obstruction, or expansion of intraluminal gas due to changes in atmospheric pressure.

Clinical manifestations of intestinal gas

Belching

Belching or eructation is defined as an audible escape of air from the esophagus or the stomach into the pharynx [8]. It may be voluntary or involuntary. Involuntary belching typically follows a meal and is caused by the release of swallowed air after gastric distention. Belching is only considered a disorder when it is excessive and becomes troublesome.
In patients with isolated symptoms of excessive belching, psychological and behavioral factors play an important role. Belching is also associated with gastroesophageal reflux disease (GERD) and functional dyspepsia [9].

Diagnostic approach

Evaluation

Belching disorder is a functional syndrome, and the diagnosis is based on a history. Observation of air swallowing provides supportive information. Patients who complain of isolated excessive belching are more likely to suffer from excessive uncontrolled supragastric belching, and from episodes of frequent belching in which they may belch up to 20 times per minute.

The presence of alarm features including weight loss, abdominal pain, dysphagia, heartburn, and regurgitation are an indication for further diagnostic evaluation with upper endoscopy and/or imaging (eg, abdominal computed tomography (CT) scan).

\section{Diagnostic criteria for functional belching}

According to the Rome IV criteria, belching disorder is defined as bothersome (ie, severe enough to impact usual activities) belching from the esophagus or stomach more than three days a week [10]. These criteria should be fulfilled for the last three months with symptom onset at least six months before diagnosis.

\section{Management}

- Education, treatment of associated disorders, and behavioral therapy: Management includes education to decrease air swallowing and reassurance that belching is a benign condition. Specific behavioral measures include discontinuation of gum chewing, smoking, drinking carbonated beverages, and gulping foods and liquids. In patients with underlying depression or anxiety, treatment should be initiated [11]. Patients with co-existing acid reflux may require acid suppressive therapy for management of GERD.

Successful treatment of excessive belching by a therapist (eg, cognitive behavioral therapist or speech therapist) with special training in diaphragmatic breathing techniques has been associated with a reduction in symptoms in small observational studies [12,13].

- Reflux inhibitors for refractory symptoms: Baclofen (10 mg three times daily) by reducing transient lower esophageal sphincter relaxations and centrally suppressing the swallowing rate, may decrease both supragastric and gastric belching [14]. 


\section{Flatulence}

The volume of gas passed per rectum varies from about $50 \mathrm{v}$ to $1500 \mathrm{~mL}$ per day [15]. The frequency of flatus released varie between 10 and 20 times per day in healthy subjects [16]. Mos individuals who report excessive flatulence fall within this range.

\section{Diagnostic evaluation}

Evaluation of the patient complaining of flatulence should be guided by the history and physical examination. A dietary history should be obtained with a focus on gas-producing foods and beverages.

\section{Alarm features}

Alarm features in patients with flatulence include the following:

- Nocturnal abdominal pain

- Weight loss

- Hematochezia

- $\quad$ Systemic symptoms including weight loss or fever

- Diarrhea or steatorrhea

- Vomiting

- Severe abdominal tenderness, organomegaly, or succussion splash on physical examination.

New onset of flatulence associated with alarm symptoms such as diarrhea and abdominal pain should be evaluated for malabsorption. Stool examination for fat and Giardia, breath tests for small intestinal and lactose intolerance, celiac serology, and endoscopic evaluation. In the absence of alarm features, additional evaluation is not required.

Management

Several measures that can be undertaken to reduce flatulence include:

- $\quad$ Treatment of the underlying cause - In individuals with lactose intolerance, management includes lactose restriction and the use of enzyme preparations that are taken orally with lactose-containing foods. In patients with small bowel bacterial overgrowth, treatment is with antibiotic therapy

- Dietary modification - Patients should be advised to avoid gas-producing foods (eg, cabbage, onions, broccoli, brussel sprouts, wheat, and potatoes). In patients without a significant improvement despite exclusion of gas-producing foods, a diet low in fermentable oligosac- charides, disaccharides, monosaccharides, and polyols (FODMAPs) $[17,18]$.

- Other interventions - Other interventions that have not consistently demonstrated efficacy in the prevention or management of flatulence include anti-flatulence medications (eg, simethicone and activated charcoal) and alpha-galactosidase enzyme preparation $[19,20]$.

Bismuth subsalicylate reduces the odor arising from hydrogen sulfide as well as other pungent components of flatus [21].

Abdominal bloating and distension

Bloating refers to a sensation of abdominal fullness, pressure, or a sensation of trapped gas, whereas distention is a measureable increase in abdominal girth. Bloating and distension have been reported in 20 to 30 percent of the general population and in up to 96 percent of patients with irritable bowel syndrome (IBS) [22-24].

\section{Evaluation}

Bloating and distension may be caused by organic disease or other functional gastrointestinal disorders. A clinical diagnosis of functional bloating/distension requires the fulfillment of symptom-based diagnostic criteria and a limited evaluation to exclude underlying organic disease.

\section{Diagnostic criteria for functional bloating/distension}

Rome IV criteria for establishing the diagnosis of functional bloating include both of the following (for at least three months with symptom onset at least six months prior to diagnosis) [25].

- $\quad$ Recurrent bloating or distension, on average, at least one day per week; abdominal bloating and/or distension predominates over other symptoms

- Insufficient criteria for a diagnosis of IBS, functional constipation, functional diarrhea, or postprandial distress syndrome.

Mild pain related to bloating may be present as well as minor bowel movement abnormalities.

\section{Management}

- Dietary and lifestyle modification: Approach to treatment of abdominal bloating and distension is similar to that used in patients with IBS. Patients are advised to avoid foods that increase flatulence (eg, beans, onions, celery, carrots, raisins, bananas, apricots, prunes, Brus- 
sels sprouts, wheat germ, pretzels, and bagels). We suggest a diet low in FODMAPs in patients with persistent symptoms despite exclusion of gas-producing foods. Other dietary modifications that may be helpful include restriction of carbonated drinks. Mild exercise and erect posture may improve bloating in some patients [26-28].

- $\quad$ Treatment of the underlying cause: In patients with small intestinal bacterial overgrowth antibiotic treatment is indicated. In individuals with lactose intolerance, management includes lactose restriction and the use of oral enzyme preparations with lactose-containing foods. Anticholinergic agents, opiates, and calcium blockers should be avoided because of their effects on gut motility

- Biofeedback: In patients with functional abdominal distension, biofeedback may decrease distension [29].

- Other therapies: Other therapies lacking clear evidence of efficacy in decreasing bloating/distension include antifoaming agents (eg, simethicone), adsorbents (eg, activated charcoal), and probiotics [30]. Although probiotics have been associated with an improvement in symptoms in patients with IBS, the magnitude of benefit and the most effective species and strain are uncertain.

\section{Conflict of Interest}

None.

\section{Bibliography}

1. Bedell Gn., et al. "Measurement of the volume of gas in the gastrointestinal tract; values in normal subjects and ambulatory patients". Journal of Clinical Investigation 35.3 (1956): 336-345.

2. Chitkara DK., et al. "Aerophagia in adults: a comparison with functional dyspepsia". Alimentary Pharmacology and Therapeutics 22 (2005): 855-858.

3. Levitt MD. "Intestinal gas production". Journal of the American Dietetic Association 60.6 (1972): 487-490.

4. Steggerda FR. "Gastrointestinal gas following food consumption". Annals of the New York Academy of Sciences 150.1 (1968): 57-66.

5. Levitt MD. "Production and excretion of hydrogen gas in man". The New England Journal of Medicine 281.3 (1969): 12-127.

6. Olesen M., et al. "Intestinal transport and fermentation of resistant starch evaluated by the hydrogen breath test". European Journal of Clinical Nutrition 48.10 (1994): 692-701.
7. Florin TH and Woods HJ. "Inhibition of methanogenesis by human bile”. Gut 37.3 (1995): 418-421.

8. Mcnally Ef., et al. "Effects of Gastric Distension With Air". Gastroenterology 46 (1964): 254-259.

9. Hemmink GJ., et al. "Supragastric belching in patients with reflux symptoms". The American Journal of Gastroenterology 104.8 (2009): 1992-1997.

10. Stanghellini V., et al. "Gastroduodenal Disorders". Gastroenterology 150.6 (2016): 1380-1392.

11. Disney B and Trudgill N. "Managing a patient with excessive belching". Frontline Gastroenterology 5.2 (2014): 79-83.

12. Katzka DA. "Simple office-based behavioral approach to patients with chronic belching". Diseases of the Esophagus 26.6 (2013): 570-573.

13. Hemmink GJ., et al. "Speech therapy in patients with excessive supragastric belching--a pilot study". Neurogastroenterology and Motility 22.1 (2010): 24-28.

14. Blondeau K., et al. "Baclofen improves symptoms and reduces postprandial flow events in patients with rumination and supragastric belching". Clinical Gastroenterology and Hepatology 10.4 (2012): 379-384.

15. Tomlin J., et al. "Investigation of normal flatus production in healthy volunteers". Gut 32.6 (1991): 665-669.

16. Olsson S., et al. "Relationship of gaseous symptoms to intestinal gas production: Symptoms do not equal increased production". Gastroenterology 108.4 (1995): A28.

17. de Roest RH., et al. "The low FODMAP diet improves gastrointestinal symptoms in patients with irritable bowel syndrome: a prospective study". International Journal of Clinical Practice 67.9 (2013): 895-903.

18. Halmos EP., et al. "A diet low in FODMAPs reduces symptoms of irritable bowel syndrome". Gastroenterology 146.1 (2014): 67-75.

19. Jain NK., et al. "Activated charcoal, simethicone, and intestinal gas: a double-blind study". Annals of Internal Medicine 105.1 (1986): 61-62.

20. Ganiats TG., et al. "Does Beano prevent gas? A double-blind crossover study of oral alpha-galactosidase to treat dietary oligosaccharide intolerance". The Journal of family practice 39.5 (1994): 441-445. 
21. Suarez FL., et al. "Bismuth subsalicylate markedly decreases hydrogen sulfide release in the human colon". Gastroenterology 114.5 (1998): 923-929.

22. Longstreth GF., et al. "Functional bowel disorders". Gastroenterology 130.5 (2006): 1480-1491.

23. Talley NJ., et al. "Identification of distinct upper and lower gastrointestinal symptom groupings in an urban population". Gut 42.5 (1998): 690-695.

24. Tuteja AK., et al. "Abdominal bloating in employed adults: prevalence, risk factors, and association with other bowel disorders". The American Journal of Gastroenterology 103.5 (2008): 1241-1248.

25. Mearin F., et al. "Bowel Disorders". Gastroenterology (2016): 2225.

26. Villoria A., et al. "Physical activity and intestinal gas clearance in patients with bloating". The American Journal of Gastroenterology 101.11 (2006): 2552-2557.

27. Sullivan SN. "Functional abdominal bloating with distention". ISRN Gastroenterol (2012): 721820.

28. Schmulson M and Chang L. "Review article: the treatment of functional abdominal bloating and distension". Alimentary Pharmacology and Therapeutics 33.10 (2011): 1071-1086.

29. Barba E., et al. "Abdominothoracic mechanisms of functional abdominal distension and correction by biofeedback". Gastroenterology 148.4 (2015): 732-739.

30. Friis H., et al. "Effect of simethicone on lactulose-induced $\mathrm{H} 2$ production and gastrointestinal symptoms". Digestion 49.4 (1991): 227-230.

Volume 2 Issue 7 September 2019

(C) All rights are reserved by Premashis Kar and Sushil

Kumar Sharma.

Citation: Premashis Kar and Sushil Kumar Sharma. “Title of the Article-Intestinal Gas”. Acta Scientific Gastrointestinal Disorders 2.7 (2019): 16-20. 